\title{
Les Centres de collaboration nationale du Canada : Faciliter la prise de décisions informées par des données probantes en matière de santé publique
}

\author{
Alejandra Dubois ${ }^{1 *}$, Mélanie Lévesque $^{1}$
}

\section{Résumé}

Bien que la prise de décisions informées par des données probantes soit essentielle à la protection de la santé publique, elle s'avère complexe dans la pratique, car on assiste en permanence à un foisonnement de données probantes et à l'apparition de nouveaux problèmes auxquels les professionnels de la santé publique doivent faire face aux échelons local, régional et national. C'est notamment par l'entremise de ses six Centres de collaboration nationale $(\mathrm{CCN})$ en santé publique que le Canada s'est attaqué à ces obstacles persistants. Les $\mathrm{CCN}$ en santé publique ont été créés afin de promouvoir et faciliter l'utilisation des connaissances issues de la recherche scientifique et des autres savoirs dans le but de renforcer les pratiques, les programmes et les politiques de santé publique au Canada. Les CCN repèrent les lacunes en matière de connaissances, favorisent le réseautage entre les secteurs et les juridictions et fournissent aux acteurs du système de santé publique un éventail de ressources et de services d'application des connaissances éclairés par des données probantes. Chaque centre est hébergé au sein d'une université ou d'un organisme gouvernemental répartis à travers le Canada, et se concentre sur un domaine prioritaire particulier de la santé publique : déterminants de la santé; santé environnementale; politiques publiques favorables à la santé; santé autochtone; maladies infectieuses; outils et méthodes d'application des connaissances. Depuis leur création en 2005, les CCN ont été soumis à deux évaluations fédérales, dont les résultats ont clairement démontré leur contribution significative à la prise de décisions informées par des données probantes en santé publique au Canada, et ont contribué à mettre en lumière des possibilités de croissance pour le futur. Les CCN permettent de jeter un pont solide entre les données probantes, les politiques et les pratiques, mais aussi de faciliter la mise en œuvre des données probantes dans des contextes souvent complexes.

Citation proposée : Dubois A, Lévesque M. Les Centres de collaboration nationale du Canada : Faciliter la prise de décisions informées par des données probantes en santé publique. Relevé des maladies transmissibles au Canada 2020;46(2/3):35-9. http//doi.org/10.14745/ccdr.v46i23a02f

Mots-clés : santé publique, réseaux, synthèse des connaissances, application des connaissances, équité en santé, déterminants de la santé, pratique éclairée par des données probantes, prise de décisions éclairées par des données probantes

\section{Introduction}

L'une des caractéristiques essentielles de la santé publique au Canada et dans le monde est l'accent mis sur la prise de décisions informées par des données probantes (PDIDP). Puisque de nouvelles connaissances sont continuellement générées, il est nécessaire, en santé publique, de continuer à synthétiser ces connaissances dans le contexte de ce qui est déjà connu, de les intégrer dans l'élaboration et la mise en œuvre des politiques et des pratiques, et d'évaluer leur application pour garantir l'atteinte des résultats escomptés. Bien que la PDIDP soit essentielle à la santé publique, elle s'avère complexe dans la pratique. L'Organisation mondiale de la Santé a reconnu que la mise en œuvre d'interventions informées par les données probantes était « l'un des plus grands défis... [pour] la communauté mondiale de la santé »(1). Les obstacles auxquels le Canada doit faire face en matière de santé publique sont notamment les suivants : 
1. Les décideurs n'ont pas forcément accès aux nouvelles données probantes à mesure que celles-ci deviennent disponibles

2. Tous les professionnels de la santé publique n'ont pas les connaissances, les compétences ou les ressources nécessaires pour entreprendre des activités de synthèse et d'application des connaissances adaptées aux besoins des décideurs provenant de divers contextes

3. Les organisations de santé publique ne possèdent pas nécessairement pas l'infrastructure nécessaire pour appuyer ces activités (2)

C'est notamment par la création de six Centres de collaboration nationale (CNN), collectivement désignés sous le nom de " programme des Centres de collaboration nationale en santé publique " (CCNSP; www.ccnsp.ca), que le Canada s'est attaqué à ces obstacles persistants. Les CCN ont été établis en 2005 dans le cadre de l'engagement du gouvernement fédéral pour le renouvellement et le renforcement de la santé publique au Canada (3). Ils visent à promouvoir et faciliter l'utilisation des connaissances issues de la recherche scientifique et des autres savoirs dans le but de renforcer les pratiques, les programmes et les politiques de santé publique au Canada. Les CCN ont été décrits comme un réseau unique de " courtiers de connaissances » (4) qui, ensemble, « repèrent les lacunes en matière de connaissances, favorisent le réseautage et fournissent aux acteurs du système de santé publique un éventail de ressources informées par les données probantes [...] et de services d'application des connaissances » (5).
Il s'agit d'un défi de taille. Les CCN s'acquittent de leur mission en favorisant la collaboration et le réseautage entre différents intervenants et en mette à profit l'expertise régionale, nationale et internationale. Ils travaillent avec une grande variété d'organisations et à travers les juridictions afin de créer des occasions d'apprendre les uns des autres et de favoriser la collaboration. Par exemple, les CCN collaborent avec le Conseil du Réseau pancanadien de santé publique, le réseau formel chargé de faire le lien entre les treize gouvernements provinciaux et territoriaux et l'Agence de la santé publique du Canada, et de rendre compte à la Conférence des sous-ministres fédéral, provinciaux et territoriaux de la Santé (6).

Cet article a pour but de décrire les activités des $\mathrm{CCN}$, de mettre en lumière leurs priorités et domaines d'interventions actuels et futurs et de préciser la manière dont ils intègrent les données probantes et les autres systèmes de connaissances dans les politiques et les pratiques.

\section{Les Centres de collaboration nationale en santé publique}

Financés par l'Agence de la santé publique du Canada et répartis d'un bout à l'autre du Canada (figure 1), les six $\mathrm{CCN}$ se concentrent chacun sur un domaine prioritaire particulier en santé publique : déterminants de la santé; santé environnementale; politiques publiques favorables à la santé; santé autochtone; maladies infectieuses; outils et méthodes

Figure 1 : Les Centres de collaboration nationale en santé publique

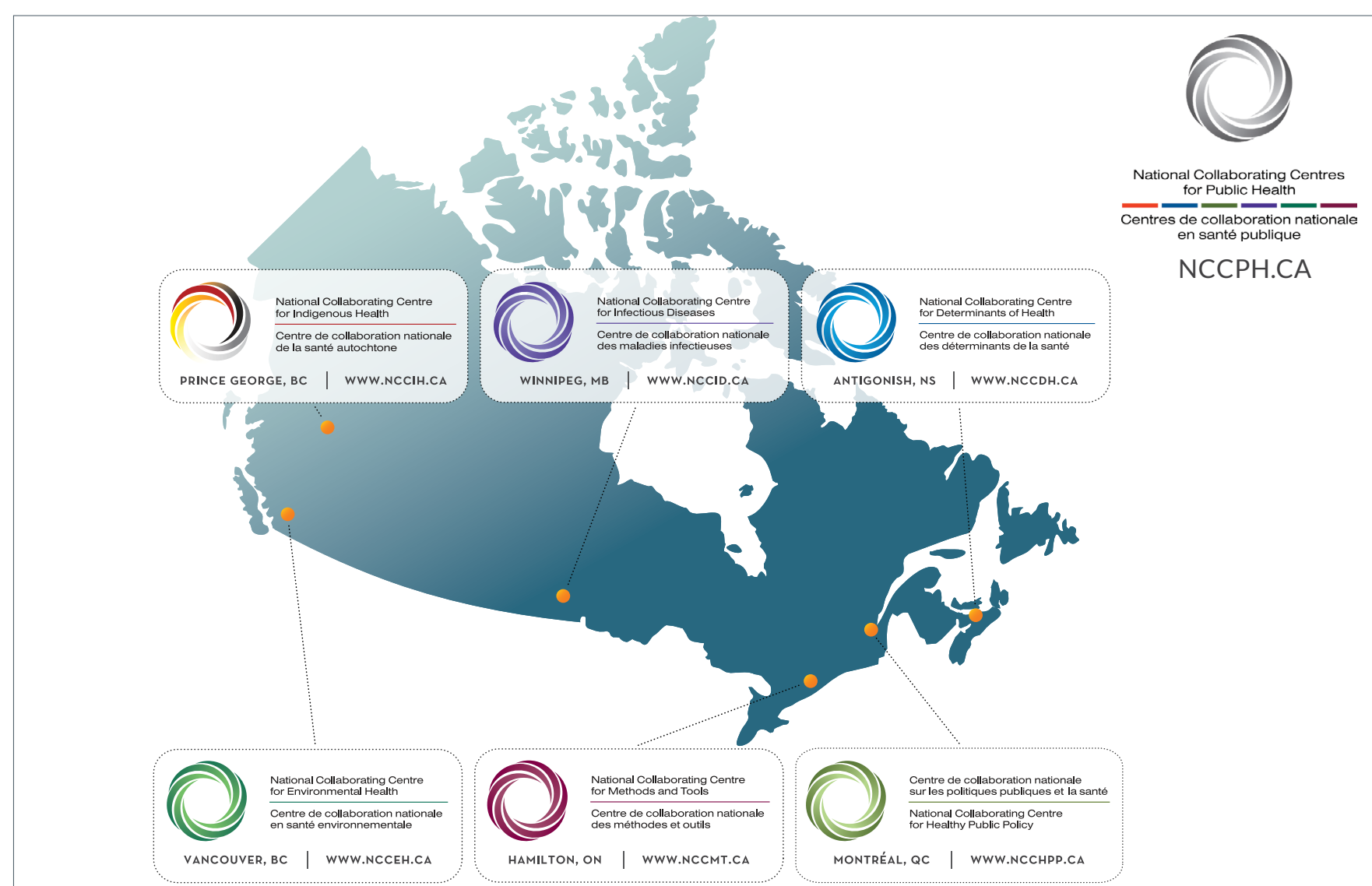


d'application des connaissances. Chaque CCN est hébergé au sein d'une université ou d'un organisme gouvernemental.

Les CCN synthétisent et diffusent des données probantes et des connaissances de haute qualité, favorisent la collaboration entre les différentes parties prenantes, et encouragent les professionnels de la santé publique, les responsables de politiques et les décideurs à utiliser les connaissances informées par des données probantes pour améliorer l'état de santé des Canadiens. Les CCN transforment les connaissances issues de la recherche scientifique et des autres savoirs en " produits de connaissance " adaptés à des publics et à des contextes spécifiques, et disponibles dans les deux langues officielles. Ces produits comprennent notamment des documents d'orientation, des rapports, des revues de données probantes, des fiches d'information et des études de cas.

Chaque CCN dispose d'un conseil consultatif d'experts pour définir les orientations et prodiguer des conseils stratégiques. Les priorités sont établies au moyen d'une grande variété de stratégies, notamment l'organisation de rencontres nationales, le soutien et la participation à des réseaux et des comités, la conduite d'enquêtes, la réalisation d'analyses environnementales et de revues de la littérature, la réalisation d'entrevues auprès des principaux répondants, l'évaluation des activités et des ressources, la conclusion de partenariats avec les dirigeants et les organismes autochtones et la collaboration avec tous les ordres de gouvernement. Comme indiqué dans un document récent que les CCNSP ont présenté au Conseil du Réseau pancanadien de santé publique (document non publié, Centres de collaboration nationale en santé publique, 2019), les priorités actuelles, les stratégies et les principaux domaines d'intervention de chaque $\mathrm{CCN}$ sont décrits dans la section suivante.

\section{$\mathrm{CCN}$ des déterminants de la santé}

Le CCN des déterminants de la santé (CCNDS) est hébergé à I'Université St Francis Xavier à Antigonish, en Nouvelle-Écosse. Le CCNDS se concentre sur trois domaines prioritaires: s'attaquer aux causes structurelles des inégalités, soutenir la « culture de l'équité » en santé publique, et promouvoir l'adoption d'actions sur les facteurs qui influencent l'équité en santé, soit les déterminants sociaux de la santé. Les stratégies établies pour y parvenir sont notamment les suivantes : faire de l'équité en santé un objectif explicite des organisations, des programmes et des politiques, promouvoir les rôles de santé publique visant à renforcer l'équité en santé, soutenir l'utilisation des connaissances et des pratiques prometteuses axées sur l'équité, faciliter le réseautage et l'échange de connaissances et influencer les pratiques d'application des connaissances pour soutenir l'adoption d'actions sur l'équité en santé.

\section{$\mathrm{CCN}$ en santé environnementale}

Le CCN en santé environnementale (CCNSE) est hébergé au Centre de contrôle des maladies de la Colombie-Britannique à Vancouver, en Colombie-Britannique. Le CCNSE se concentre sur trois priorités. La première consiste à sensibiliser et mieux faire comprendre 1) les risques et les bénéfices actuels et émergents pour l'environnement et 2) les moyens d'atténuer ces risques et d'optimiser les bénéfices. La deuxième consiste à traduire et à mettre en lumière les recherches qui informent les pratiques efficaces en matière de santé environnementale. La troisième consiste à mettre à profit l'expérience cumulative des praticiens en santé environnementale de tout le Canada pour éclairer les pratiques réputées efficaces et adaptées au caractère évolutif de la santé publique. Les domaines prioritaires sont notamment 1) les environnements naturels et bâtis, 2) les changements climatiques, la préparation et la réponse en cas d'urgence, et 3) les ressources destinées à faciliter les inspections sanitaires, la protection de la santé publique et l'élaboration des politiques.

\section{CCN sur les politiques publiques et la santé} Le CCN sur les politiques publiques et la santé (CCNPPS) est hébergé à I'Institut national de santé publique du Québec à Montréal, au Québec. Le CCNPPS se concentre sur trois priorités. La première consiste à soutenir l'amélioration des compétences et des capacités organisationnelles en analyse des politiques. La deuxième consiste à faciliter la mise en œuvre d'approches intersectorielles afin de promouvoir des politiques publiques favorables à la santé. La troisième consiste à élaborer des approches en matière de politiques en ce qui concerne les enjeux émergents de santé publique. Les domaines prioritaires sont notamment l'analyse des politiques, la Santé dans toutes les politiques, l'évaluation d'impact sur la santé, les changements climatiques, la santé mentale et le bien-être des populations, l'éthique en santé publique, les inégalités de santé et le partage des connaissances.

\section{$\mathrm{CCN}$ de la santé autochtone}

Le CCN de la santé autochtone (CCNSA) est hébergé à I'Université de Northern British Columbia à Prince George, en Colombie-Britannique. Le CCNSA se concentre sur deux priorités. La première consiste à mieux comprendre et utiliser les données probantes issues des savoirs autochtones sur la santé des Premières Nations, des Inuits et des Métis tout au long de leur vie, et ce, pour faciliter la prise de décisions sur les politiques, les pratiques et les programmes de santé publique. La deuxième consiste à encourager les partenariats, la collaboration et le réseautage entre les secteurs et les juridictions afin de mobiliser les données probantes issues des savoirs autochtones et d'améliorer l'équité en santé des populations autochtones. Les axes du CCNSA en matière de santé sont notamment les priorités émergentes de santé publique, les déterminants sociaux de la santé, la santé des enfants, des jeunes et des familles, et les connaissances autochtones et la santé publique. 
est de choisir les enjeux sur lesquels travailler, les partenaires à impliquer, ainsi que les lieux et les modalités de synthèse et d'application des connaissances. Le secteur de la santé publique regroupe en effet une main-d'œuvre interdisciplinaire variée, un large éventail de programmes et de services et un vaste réseau de partenaires et de parties prenantes qui s'entrecroisent, notamment des membres des communautés et tous les ordres de gouvernement.

Pour garantir l'efficacité du programme des CCNSP, deux évaluations fédérales ont été menées : la première ciblait la période allant de 2008 à début 2014 (7), et la seconde, la période allant de 2014 à septembre 2018 (8). Selon les conclusions du dernier rapport d'évaluation, les CCN sont des sources incontournables d'information crédibles sur de nombreux enjeux de santé publique, générant une grande variété de produits d'application des connaissances de haute qualité. Le rapport a mis en évidence de nombreux exemples où les $\mathrm{CCN}$ ont contribué à la prise de décisions et à l'élaboration de politiques dans le domaine de la santé publique. II y est également précisé que la « capacité des $\mathrm{CCN}$ à collaborer sur différentes initiatives et à réseauter avec différents partenaires du système de santé publique était considérée comme l'une de leurs compétences les plus précieuses » (8).

L'évaluation réalisée en 2018 contient également de nombreux exemples où les $\mathrm{CCN}$ ont contribué à aborder les nouveaux enjeux de santé publique. Elle souligne néanmoins la nécessité de gagner en flexibilité pour faire face à des enjeux émergents, ainsi que l'intérêt potentiel d'une plus grande collaboration avec l'Agence de la santé publique du Canada pour mettre à profit les connaissances, les ressources et les réseaux respectifs.

Les priorités, les stratégies et les domaines d'intervention actuels des $\mathrm{CCN}$ s'alignent à plusieurs des tendances macro actuelles en santé publique : changements climatiques, Santé dans toutes les politiques, déterminants structurels des inégalités de santé, mégadonnées et transitions démographiques (comme les populations migrantes et mobiles) (9). Le travail des CCN concorde également avec la nouvelle approche "Public Health 3.0 » préconisée par le ministère de la Santé et des Services sociaux des États-Unis, laquelle met l'accent sur la " collaboration intersectorielle et l'adoption de mesures à l'échelle de l'environnement, de la politique et des systèmes pour influer directement sur les déterminants sociaux de la santé » (10).

L'Agence de la santé publique du Canada reconnaît la contribution des $\mathrm{CCN}$ et réaffirme son engagement à renforcer la capacité de santé publique par la science, le savoir et la PDIDP. Les CNC sont un pilier fondamental de l'infrastructure de santé publique du Canada et un atout très précieux à protéger et à bien utiliser.
Près de quinze ans après leur création, les $\mathrm{CCN}$ ont démontré leur capacité à répondre aux besoins de la santé publique avec des données probantes, des systèmes de connaissances et le développement de réseaux. L'un de leur plus gros défis 


\section{Conclusion}

Il existe un besoin continu en ce qui concerne les services d'application des connaissances pour rendre les données probantes accessibles et utiles aux professionnels et aux organisations de santé publique, ainsi qu'à tous les ordres de gouvernement, pour faire progresser les priorités nationales en matière de santé publique. De plus, « dans le système de santé multijuridictionnel du Canada, il demeure essentiel de renforcer les réseaux qui y sont établis. Les CCN occupent une niche unique, axée sur l'application pratique des connaissances et des données probantes pour appuyer les professionnels de la santé du pays » (8). Les CCN continuent à jouer un rôle crucial dans le domaine de la santé publique au Canada, et ce, en repérant les lacunes en matière de connaissances et en jetant des ponts entre les données probantes, les politiques et les pratiques. Les CCN ont su démontrer leur capacité à faciliter la mise en œuvre des données probantes dans des contextes souvent complexes, et continueront à assumer un rôle de premier plan dans le système de santé publique du Canada.

\section{Déclaration des auteurs}

A. D. - Conception originale, examen des versions préliminaires et revision finale

M. L. - Contribution à la conception, appui technique et examen des versions préliminaires

\section{Conflit d'intérêts}

Aucun.

\section{Remerciements}

Nous tenons à remercier les principaux membres de chaque Centre de collaboration nationale en santé publique d'avoir examiné les versions préliminaires de cet article et d'avoir apporté leurs contributions pendant la période des vacances. Nous remercions également $D^{\text {re }}$ Patricia Huston pour sa contribution à la structure initiale et à la conception de ce manuscrit.

\section{Références}

1. Peters DH, Tran NT, Adam T. La recherche sur la mise en œuvre en santé : Guide pratique. Alliance pour la recherche sur les politiques et les systèmes de santé, Organisation mondiale de la Santé. Genève (CH) : OMS; 2013. https:// www.who.int/alliance-hpsr/resources/Implementation_ Research_FR2.PDF?ua $=1$
2. Peirson L, Ciliska D, Dobbins M, Mowat D. Building capacity for evidence informed decision making in public health: a case study of organizational change. BMC Public Health 2012 Feb;12:137. DOl PubMed

3. Medlar B, Mowat D, Di Ruggiero E, Frank J. Introducing the National Collaborating Centres for Public Health. CMAJ 2006 Aug;175(5):493-4. DOl PubMed

4. McAteer J, Di Ruggiero E, Fraser A, Frank JW. Bridging the academic and practice/policy gap in public health: perspectives from Scotland and Canada. J Public Health (Oxf) 2019 Sep;41(3):632-7. DOI PubMed

5. Centres de collaboration nationale en santé publique. À propos. (Accédé 2020-01-02). https://ccnsp.ca/about-us/

6. Réseau pancanadien de santé publique. À propos du Réseau pancanadien de santé publique. RSP (Accédé 2019-12-31). http://www.phn-rsp.ca/network-fra.php

7. Santé Canada et de l'Agence de la santé publique du Canada. Évaluation du programme des Centres de collaboration nationale en santé publique de 2008-2009 à 2013-2014. Ottawa (ON) : ASPC (Accédé 2020-01-02). https://www.canada.ca/fr/sante-publique/organisation/ mandat/a-propos-agence/bureau-evaluation/rapportsevaluation/evaluation-programme-centres-collaborationnationale-sante-publique-2008-2009-a-2013-2014.html

8. Santé Canada et de l'Agence de la santé publique du Canada. Évaluation du programme des Centres de collaboration nationale en santé publique de 2014-2015 à 2018-2019. Ottawa (ON) : ASPC; p. 33. (Accédé 2020-0102). https://www.canada.ca/fr/sante-publique/organisation/ transparence/rapports-gestion/evaluation/2014-2015-20182019-rapport-evaluation-programme-centres-collaborationnationale-sante-publique.html

9. Erwin PC, Brownson RC. Macro Trends and the Future of Public Health Practice. Annu Rev Public Health 2017 Mar;38:393-412. DOl PubMed

10. DeSalvo KB, O'Carroll PW, Koo D, Auerbach JM, Monroe JA. Public Health 3.0: time for an Upgrade. Am J Public Health 2016 Apr;106(4):621-2. DOI PubMed 